\title{
Role of uterine artery Doppler in assessment of unexplained infertility
}

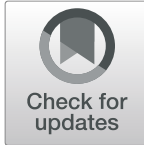

Carmen Ali zarad ${ }^{1 *}$, Mohamed Hafez Mohamed² and Waleed Said Abo Shanab

\begin{abstract}
Background: The study aimed to compare the values of uterine artery Doppler indices including resistance index (RI), pulsatility index (PI), and systolic/diastolic (S/D) ratios in fertile female and female with unexplained infertility and investigate their association with unexplained infertility. This study included 70 women divided into two groups. Study group included 40 women with unexplained infertility and control group included 30 fertile women.

Results: The mean ages of study and control groups were 26.9 and 28.5 years respectively. In infertile group, the mean values of uterine arteries resistance index (RI), pulsatility index (PI), and systolic/diastolic (S/D) ratios were 0.9, 2.9 , and 8.0 respectively. In the fertile control group, mean $\mathrm{RI}$ and PI values and S/D ratio were $0.6,1.5$, and 2.7 respectively. There was statistically significant difference as regards $\mathrm{RI}, \mathrm{PI}$, and S/D ratios between study and control groups. The best cut off values of RI, PI, and S/D ratios for predicting increased uterine blood flow impedance were more than $0.67,1.95$, and 3 respectively with sensitivity of $100 \%, 95 \%$, and $100 \%$ respectively, specificity of $96.7 \%$, $86.7 \%$, and $96.7 \%$ respectively, and diagnostic accuracy of $98.6 \%, 91.42 \%$, and $98.57 \%$ respectively.

Conclusion: Uterine artery Doppler indices mean values were higher in unexplained infertility women than fertile women. Uterine artery Doppler indices have high sensitivity and specificity for diagnosis of high uterine blood flow impedance. High uterine blood flow impedance diagnosed by uterine artery Doppler may contribute in the etiology of unexplained infertility. Uterine artery Doppler should be included in investigation of unexplained infertility.
\end{abstract}

Keywords: Resistance index, Pulsatility index, Uterine artery Doppler, Unexplained infertility

\section{Background}

Infertility is defined as an inability to conceive 12 months or more in spite of regular and non-protected sexual intercourse [1]. Many investigations are needed to detect the cause of infertility [2].

Unexplained infertility is defined as failure of couple to achieve pregnancy without presence of definite cause after 12 months of trying conception, or after 6 months in female more than or equal 35 years old [3]. Ten to twenty five percent (10-25\%) of infertile females had no definite cause for infertility and diagnosed with unexplained infertility [2].

\footnotetext{
* Correspondence: carmenali79@yahoo.com

${ }^{1}$ Diagnostic Radiology, Faculty of Medicine, Port Said University, Port Said, Egypt

Full list of author information is available at the end of the article
}

Successful implantation of embryo in the uterus depends mainly on the ovum quality and receptivity of uterine endometrium. Good endometrial growth is important factor for achievement of successful implantation. Endometrial thinning is associated with low pregnancy rates [4].

Many factors regulated the receptivity of the uterine endometrium including uterine artery blood flow and endometrial perfusion. Adequate blood flow of uterus is important for good endometrial growth and increase the endometrial receptivity [5]. Increased impedance of uterine arteries' blood flow leads to poor growth of endometrium and endometrial thinning [3].

Many studies concluded that decreased uterine artery blood flow and endometrial perfusion could be an important factor for diagnosis of unexplained infertility, 
abortion, and in vitro fertilization (IVF) failure [6]. Many studies concluded that women with unexplained infertility had higher uterine artery impedance than that of the normal fertile women so decreased uterine artery blood flow and uterine perfusion could be a cause of unexplained infertility [7].

Ultrasound is highly effective for studying the reproductive system of the female and also valuable in followup of the functional changes during spontaneous and induced menstrual cycles. Transvaginal ultrasonography has a great importance in diagnosis of causes of infertility [8].

Normally at mid-luteal phase endometrium changed from proliferative to secretory phase, blood supply of uterus is increased, uterine artery impedance is decreased, and so receptivity of the uterine endometrium increases [9].

Normally, the endometrial perfusion and blood flow of uterine artery improved significantly during the luteal phase of menstrual cycle; therefore, the use of uterine artery Doppler and measuring the uterine arterial impedance could evaluate the endometrial receptivity [10].

Assessment of uterine artery blood flow and endometrial perfusion by color Doppler can be used to detect the cause of infertility especially unexplained infertility [7].

The aim of the study is to compare the measures of uterine artery Doppler indices including the resistance index (RI), the pulsatility index (PI), and the systolic/diastolic (S/D) ratio in fertile female and female with unexplained infertility and investigate their association with unexplained infertility.

\section{Methods}

This study was performed during the period from April to September 2020 in the ultrasound outpatient clinic of our institution. The study was approved by the Research Ethic Committee (REC) of our institution.

This study included two groups of women:

Group 1 (study group): 40 women diagnosed with unexplained infertility.

Group 2 (control group): 30 fertile women that were visited the radiology clinic for ultrasound examination for any other gynecological cause.

The procedure was explained to all women who participated in the study and written informed consent was obtained from all women that were included in the study.

\section{Inclusion criteria}

- Married women in both groups.

- Age: less than 40 years old in both groups.

- No history of systemic or gynecological diseases that cause infertility in both groups.
- Women did not use intrauterine devices or oral contraception.

\section{Exclusion criteria}

- Unmarried virgin women.

- Age: more than 40 years old.

- History of systemic or gynecological diseases that cause infertility.

- Use of intrauterine device or oral contraception.

- The patient refused the consent.

In our study, all women were subjected to full history taking including age, last menstrual period, parity, and any history of medical or gynecological diseases.

All 70 women of both study and control groups underwent transvaginal ultrasound and bilateral uterine artery Doppler in the mid-luteal phase of menstrual cycle by two radiologists using Xario 200 ultrasound machine (Canon Medical System, Toshiba, Japan) with 7.5 MHz endovagianl probe

First, the uterus was imaged in sagittal plane for measurement of endometrial thickness; then, the transducer moved laterally to evaluate the uterine artery when crossing the external iliac vessels.

Three consequent similar waves of uterine artery flow were obtained after activation of Doppler wave and adjustment of insonation angle. Analysis of uterine artery Doppler waveforms was done by simple semiquantitative methods that depended on assessment of the Doppler waveform and measurement of Doppler spectral parameter including the resistance index (RI), the pulsatility index (PI), and the systolic/diastolic ratio (S/D).

For each woman in both groups Doppler spectral parameters for both uterine arteries were measured by two radiologists independently and the mean values were calculated. All Doppler measures recorded by both radiologists were convergent, and there was no any controversy in-between.

\section{Statistical analysis}

Before testing the statistical significance, normality of data was tested by Kolmogorov-Smirnov test. Nonparametric tests of significance were used for not normally distributed variables.

The Student $t$ test was used to evaluate differences in the mean value between 2 groups for normally distributed variables and Mann-Whitney test was used for not normally distributed variables.

Pearson's correlation coefficient $(r)$ test was used for correlating normally distributed data and Spearman correlation coefficient for not normally distributed variables. 
Statistical significance was considered at 95\% level of confidence (significant if $p$ value $<0.05$ ).

\section{Results}

This study included two groups: group one (study group) and group two (control group). Group one (study group) included 40 women that were diagnosed with unexplained infertility with a mean age of $26.9 \pm 3.8$ years. Group two (control group) included 30 fertile women with a mean age about $28.5 \pm 4.9$ years. As regards the mean age of both groups, there was no significance difference between fertile and infertile groups $(P=0.194)$ (Table 1).

The mean values of uterine arteries resistance index (RI) and pulsatility index (PI) were found to be $0.9 \pm$ $0.06 \mathrm{SD}$ and $2.9 \pm 0.7 \mathrm{SD}$ respectively in the unexplained infertility study group respectively. In the fertile control group, mean RI and PI values were calculated as $0.6 \pm$ $0.04 \mathrm{SD}$ and $1.5 \pm 0.4 \mathrm{SD}$ respectively. The difference between the study and the control group for RI $(p<0.001)$ and PI $(p<0.001)$ was statistically significant.

Uterine arteries systolic/diastolic (S/D) ratios and endometrial thickness mean values were found to be 8.0 \pm 3.4 SD and $10.4 \mathrm{~mm} \pm 2.4 \mathrm{SD}$ in the unexplained infertility study group respectively. For the fertile control group, mean systolic/diastolic ratio and endometrial thickness were calculated as $2.7 \pm 0.3 \mathrm{SD}$ and $12.1 \mathrm{~mm}$ $\pm 1.8 \mathrm{SD}$ respectively. The difference between the study and the control group for systolic/diastolic ratios $(p<$ $0.001)$ and endometrial thickness $(p<0.001)$ were also statistically significant.

No significant difference was observed between the participants in both groups according to age $(p>0.05)$ (Table 1).

It was found that there is no significant correlation between endometrial spiral arteries resistance index (RI), pulsatility index (PI), systolic/diastolic (S/D) ratio, endometrial thickness, and age in both the infertile and fertile groups (Tables 2 and 3 ).
Receiver operating characteristics (ROC) curve was used to define the best cut-off value for assessment of uterine artery blood flow impedance.

The best cut off value of uterine artery resistance index (RI) was 0.67 , with sensitivity of $100 \%$, specificity of $96.7 \%$, positive predictive value of $97.5 \%$, negative predictive value of $100 \%$, and diagnostic accuracy of $98.6 \%$ (Fig. 1 and Table 4).

The best cut-off value of uterine artery pulsatility index (PI) was 1.95 , with sensitivity of $95 \%$, specificity of $86.7 \%$, positive predictive value of $90.47 \%$, negative predictive value of $92.86 \%$, and diagnostic accuracy of 91.42\% (Fig. 2 and Table 4).

The best cut-off value of systolic/diastolic (S/D) ratios was 3 , with sensitivity of $100 \%$, specificity of $96.7 \%$, positive predictive value of $97.56 \%$, negative predictive value of $100 \%$, and diagnostic accuracy of $98.57 \%$ (Fig. 3 and Table 4).

\section{Discussion}

The uterine endometrium shows multiple growth phases during menstrual cycle. By the end of previous menstruation, endometrial proliferation starts during follicular phase of menstrual cycle. The endometrium continues to grow and changes from proliferative phase to secretory phase during the luteal phase of menstrual cycle. The endometrium continues to grow until sudden drop in the hormone level occur leading to menstruation [11].

The main function of endometrium is to provide a time-limited support for embryo implantation. The uterine endometrium shows resistance for embryo implantation during most phases of menstrual cycle. Endometrium achieves special receptive properties during luteal phase of menstrual cycle and this period is called implantation window [12].

The blood flow impedance of uterine and spiral arteries is changed periodically during normal menstrual cycle. The lowest blood flow impedance occurs during the mid-luteal phase of menstrual cycle. The high uterine blood flow in this stage increases the endometrial

Table 1 Comparison between the unexplained infertility group and the fertile control group according to the mean values of age, uterine arteries resistance index, pulsatility index, systolic/diastolic ratios, and endometrial thickness

\begin{tabular}{llll}
\hline & $\begin{array}{l}\text { Unexplained infertility group }(\boldsymbol{n}=40) \\
\text { Mean } \pm \text { SD }\end{array}$ & $\begin{array}{l}\text { Fertile control group }(\boldsymbol{n}=\mathbf{3 0}) \\
\text { Mean } \pm \text { SD }\end{array}$ & $\boldsymbol{p}$ value \\
\hline Age (years) & $26.9 \pm 3.8$ & $28.5 \pm 4.9$ & $0.194^{\#}$ \\
Resistance index & $0.9 \pm 0.06$ & $0.6 \pm 0.04$ & $<0.0001^{* \pi}$ \\
Pulsatility index & $2.9 \pm 0.7$ & $1.5 \pm 0.4$ & $<0.0001^{* \#}$ \\
systolic/diastolic ratio (S/D): & $8.0 \pm 3.4$ & $2.7 \pm 0.3$ & $<0.0001^{* \pi}$ \\
Endometrial thickness & $10.4 \pm 2.4$ & $12.1 \pm 1.8$ & $<0.001^{* \pi}$ \\
\hline
\end{tabular}

*Statistically Significant at $95 \%$ level of confidence

"Student's $t$ test is used

"Mann-Whitney test is used 
Table 2 Correlation between uterine arteries resistance index, pulsatility index, systolic/diastolic ratios, endometrial thickness, and age of the participants in the unexplained infertility (study) group

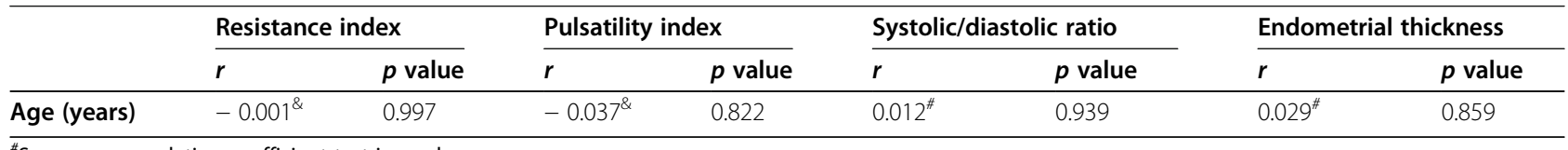

${ }^{\#}$ Spearman correlation coefficient test is used

\&Pearson's correlation coefficient test is used

receptivity and gives a good chance for embryo implantation to occur [13].

Many methods are used to evaluate the receptivity of the endometrium like endometrial biopsy and immunehistochemical analysis. Doppler ultrasonography is considered as simple non-invasive method that could be used to assess the blood flow impedance of uterine arteries and provides an indirect method to measure the receptivity of the endometrium [7].

In women with unexplained infertility, the perfusion of the endometrium in the pre-implantation stage is lower than normal despite of endometrial measurement or hormonal profile [14].

Many studies showed that in women with unexplained infertility the uterine and spiral arteries' impedance was higher than that of the fertile females and the decrease of the perfusion of uterus may be a cause of the unexplained infertility $[8,11,13]$.

Many studies evaluated the role of subendometrial blood flow impedance in the pathogenesis of unexplained infertility. In these studies, transvaginal uterine artery Doppler indices were measured in the mid-luteal phase of menstrual cycle (pre-implantation phase) in women diagnosed with unexplained infertility and compared the values with that of control fertile groups. The studies reported that in women with unexplained infertility, the pulsitility index (PI) and resistance index (RI) of uterine artery were increased significantly in women diagnosed with unexplained infertility more than fertile women $[8,13-17]$.

The most common Doppler indices used for assessment of uterine artery blood flow impedance are the resistance index (RI), the pulsatility index (PI), and the systolic/diastolic (S/D) ratio [18].

This study included two groups of women. Group 1 (study group) included 40 women diagnosed with unexplained infertility (Figs. 6, 7, 8, 9, and 10) and group 2 (control group) included 30 fertile women who visited the radiology clinic seeking for transvaginal ultrasound for any other gynecological cause (Figs. 4 and 5).

In this study, the mean age of the study group was $26.9 \pm 3.8$ years and the mean age of the control group was $28.5 \pm 4.9$ years with no significant difference between both groups $(P=0.194)$ as regards the age. These results were matched with Hashad et al.; they concluded that the mean age of women in the study group was 26 years while in the control group was 27 years [8]. ElShourbagy et al. concluded that as regards the age there was no significant difference between fertile and infertile groups [3].

In this study, the resistance index (RI) mean value was $0.9 \pm 0.06 \mathrm{SD}$ in the study (infertile) group and was $0.6 \pm 0.04 \mathrm{SD}$ in the control (fertile) group with statistically significant difference between both study and control groups. These results were matched with Hashad et al. they concluded that the mean RI values in the infertile group and the fertile group were 0.69 and 0.61 respectively and that there was a significant difference between the fertile and infertile groups as regards the mean value of resistance index (RI) [8].

In the current study, the mean value of pulsatility index (PI) in the study (infertile) group was $2.9 \pm 0.7$ $\mathrm{SD}$ and was $1.5 \pm 0.4 \mathrm{SD}$ in the control (fertile) group with statistically significant difference between both groups. These results were matched with Hashad et al. [8] and Selda et al. [15]; they concluded that there was a significant difference between the fertile and infertile groups as regards the mean value of pulsatility index (PI). Hashad et al. found that the mean values of PI in the study and control groups were 1.3 and 1.16 respectively. Selda et al. found that the mean values of PI in the study and control groups were 0.8 and 0.7 respectively [15].

Table 3 Correlation between uterine arteries resistance index, pulsatility index, systolic/diastolic ratios, endometrial thickness, and age of the participants in (fertile) control group

\begin{tabular}{|c|c|c|c|c|c|c|c|c|}
\hline & \multicolumn{2}{|c|}{ Resistance index } & \multicolumn{2}{|c|}{ Pulsatility index } & \multicolumn{2}{|c|}{ Systolic/diastolic ratio } & \multicolumn{2}{|c|}{ Endometrial thickness } \\
\hline & $r$ & $p$ value & $r$ & $p$ value & $r$ & $p$ value & $r$ & $p$ value \\
\hline Age (years) & $0.085^{\#}$ & 0.656 & $0.259^{\&}$ & 0.167 & $0.311^{\&}$ & 0.094 & -.260 & $0.166^{\#}$ \\
\hline
\end{tabular}

\#Spearman correlation coefficient test is used

\&Pearson's correlation coefficient test is used 


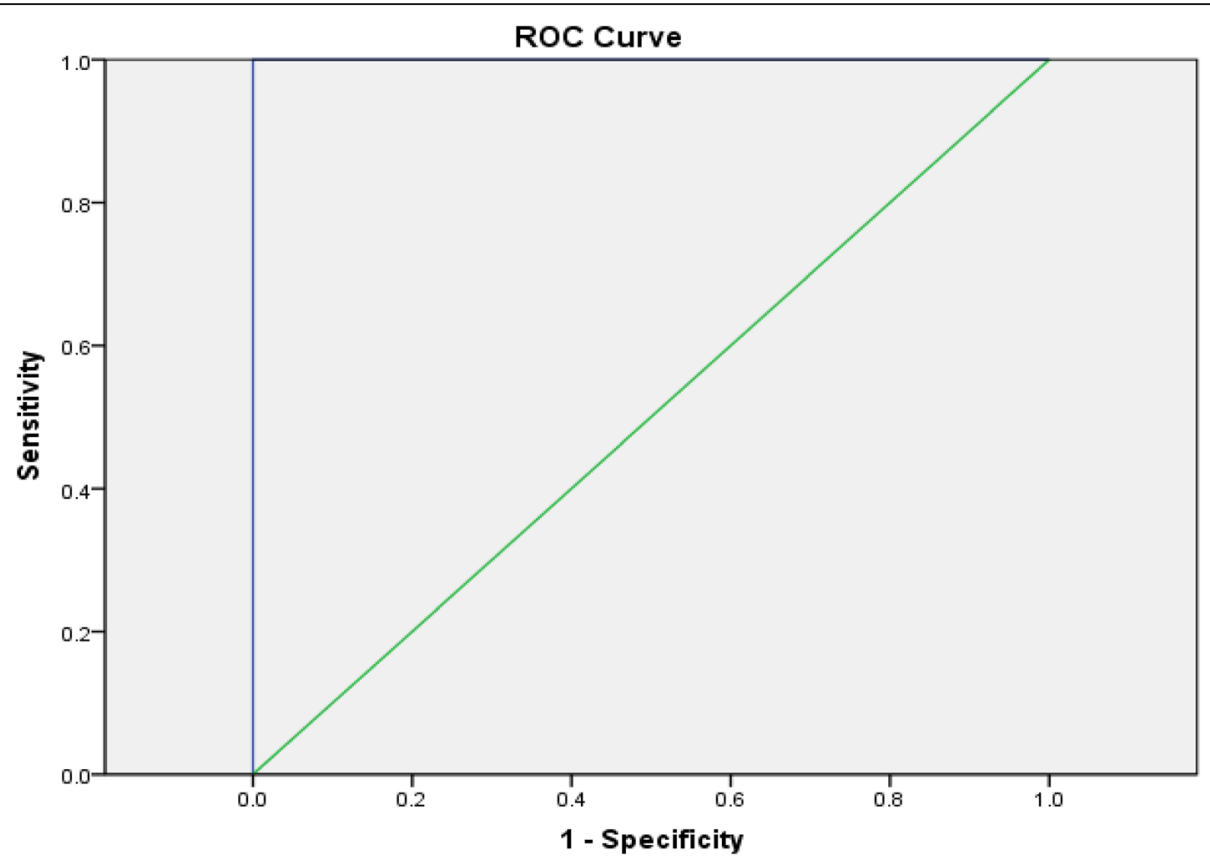

Fig. 1 Receiver-operating characteristic (ROC) curve for uterine arteries resistance index in discrimination of the unexplained infertility group and the fertile control group

This study was in agreement with Chien et al.; they concluded that resistance index (RI) and pulsatility index (PI) of uterine artery were lower in conception menstrual cycles more than in non-conception cycles [19].

Studies of Devyatova et al. found that $35 \%$ of females who underwent in vitro fertilization (IVF) with mean pulsatility index (PI) of uterine artery more than 3 failed to conceive [9].

The study of Cacciatore et al. aimed to detect the role of uterine artery blood flow impedance in the outcome of in vitro fertilization embryo transfer (IVF-ET) by measuring uterine artery RI and PI values. Their results were in agreement with our results as they concluded that the uterine artery blood flow impedance was significantly lower in the conception group than in nonconception group. They also concluded that the endometrium was not receptive and implantation did not occur when resistance index (RI) and pulsatility index

Table 4 The diagnostic performance of uterine arteries' RI, PI, and S/D ratios in discrimination of both the infertile and fertile groups

\begin{tabular}{lllllll}
\hline Item & Cut-off & Sensitivity & Specificity & PPV & NPV & Accuracy \\
\hline $\mathrm{RI}$ & $>0.67$ & $100 \%$ & $96.7 \%$ & $97.5 \%$ & $100 \%$ & $98.6 \%$ \\
$\mathrm{PI}$ & $>1.95$ & $95 \%$ & $86.7 \%$ & $90.47 \%$ & $92.86 \%$ & $91.42 \%$ \\
$\mathrm{~S} / \mathrm{D}$ & $>3$ & $100 \%$ & $96.7 \%$ & $97.56 \%$ & $100 \%$ & $98.57 \%$ \\
\hline
\end{tabular}

(PI) of uterine arteries are more than 0.72 and 1.6 respectively [20].

In this study, the mean uterine artery systolic/diastolic (S/D) ratio for the study (infertile) group was found to be $8.0 \pm 3.4 \mathrm{SD}$ and for the control (fertile) group was $2.7 \pm 0.3 \mathrm{SD}$. The difference between the study and the control group as regards systolic/diastolic (S/D) ratio was statistically significant.

The study of Ari et al. was done by using 3D power Doppler ultrasound to assess the blood flow of endometrium in order to predict the pregnancy outcome after intrauterine insemination (IUI) [21]. Ari et al. concluded that the Doppler indices measured by 3D power Doppler like resistance index (RI), pulsatility index (PI), and systolic/diastolic (S/D) ratio were useful predictors for pregnancy after IUI [21].

Khan et al. reported that pregnancy did not occur in the case of absent or reversed uterine end diastolic blood flow [22].

In the current study, the mean endometrial thickness of the study group was $10.4 \mathrm{~mm} \pm 2.4 \mathrm{SD}$ and of the control group was $12.1 \mathrm{~mm} \pm 1.8 \mathrm{SD}$. The difference between the study and the control group as regards endometrial thickness was statistically significant between both groups. This matched with the studies of Khan et al. [22], Noyes et al. [23], and Kovacs et al. [24] they reported that pregnancy rates were significantly higher in women with endometrial thickness more than $7 \mathrm{~mm}$ 


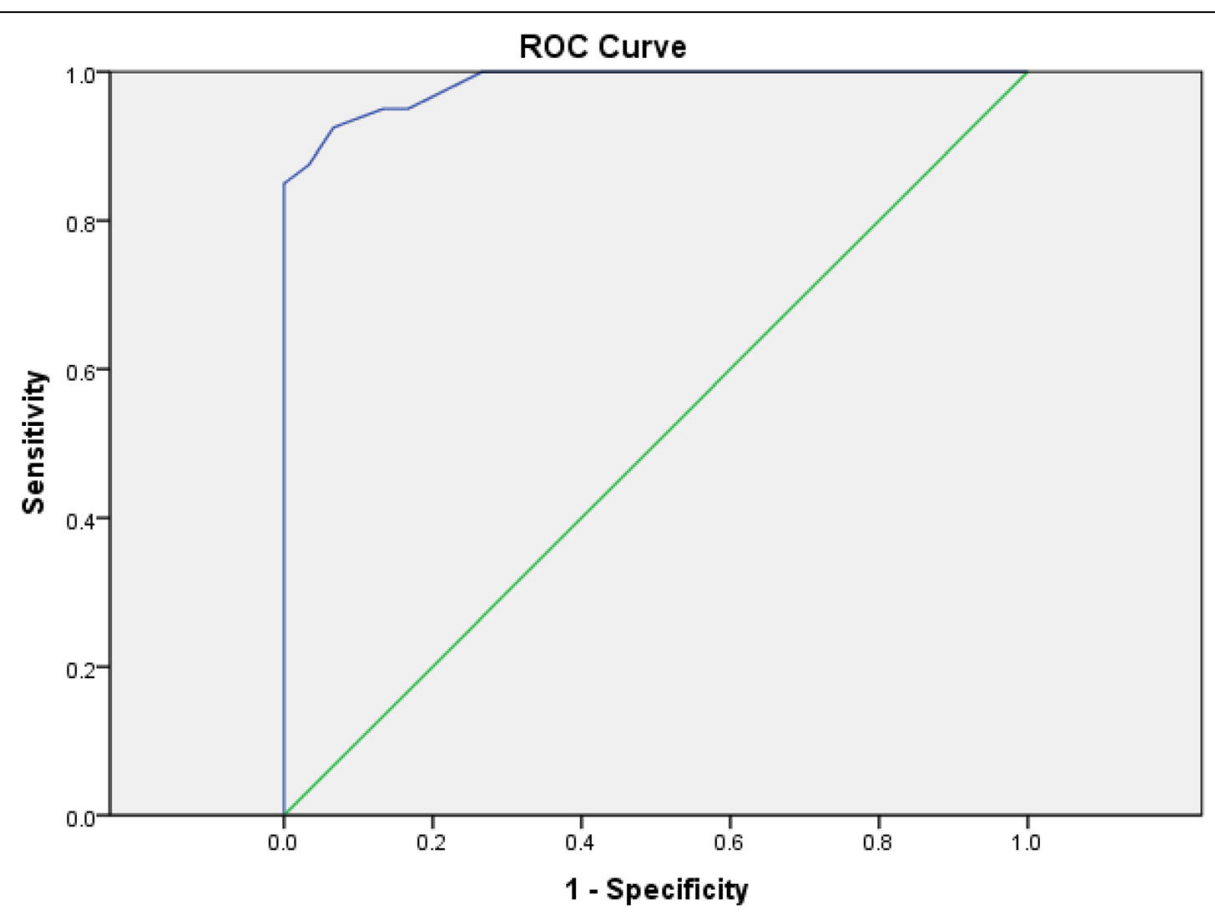

Diagonal segments are produced by ties.

Fig. 2 Receiver-operating characteristic $(\mathrm{ROC})$ curve for uterine arteries pulsatility index in discrimination of the unexplained infertility group and the fertile control group

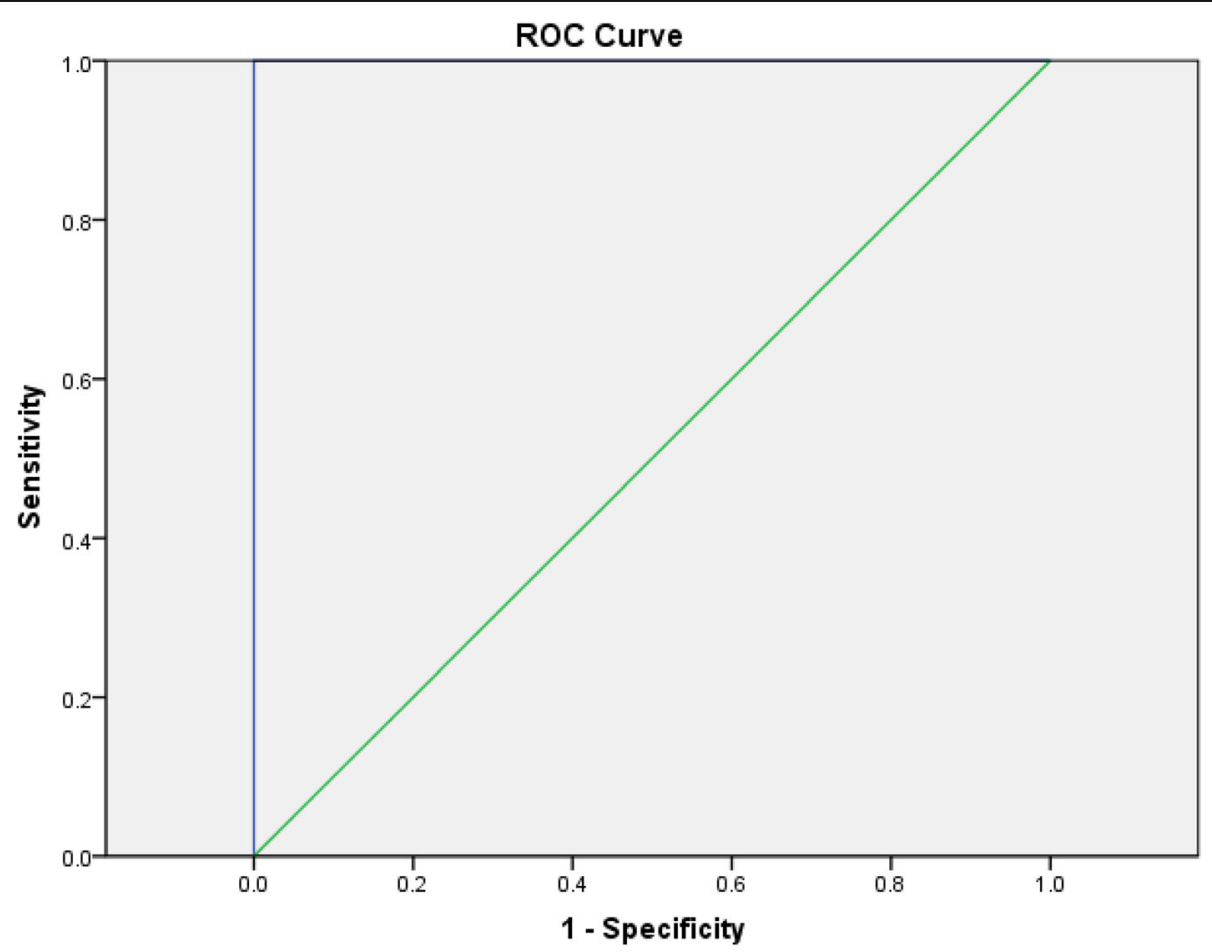

Fig. 3 Receiver-operating characteristic (ROC) curve for uterine arteries systolic/diastolic ratios in discrimination of the unexplained infertility group and the fertile control group 


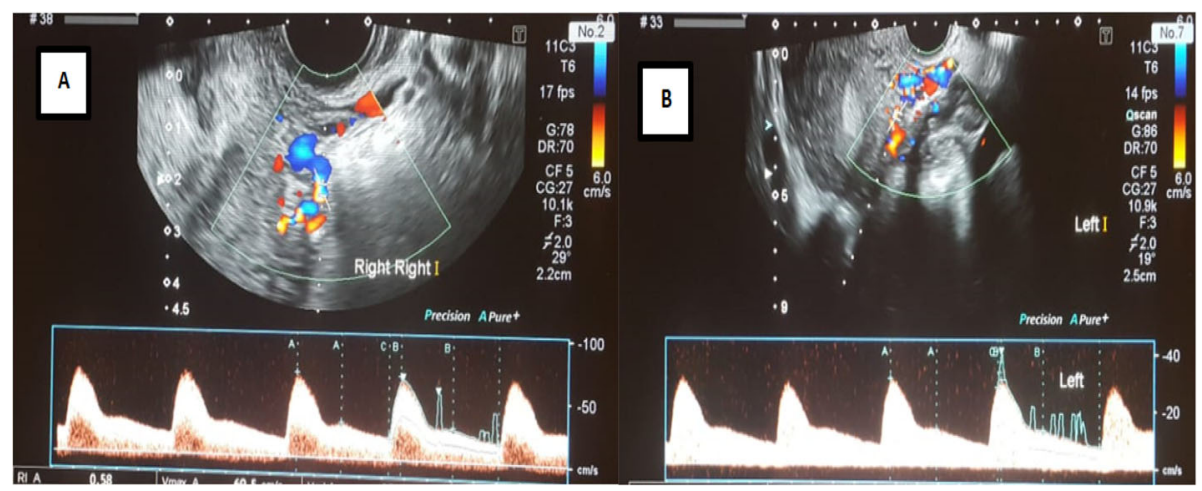

Fig. 4 a, b Transvaginal Doppler ultrasound of both uterine arteries (a right, $\mathbf{b}$ left) of fertile female (control group) aged 25 years old shows RI of right and left uterine arteries were 0.58 and 0.58 with mean RI 0.58, S/D ratios of right and left uterine arteries were 2.49 and 2.36 respectively with mean S/D ratio 2.42, and PI of right and left uterine arteries were 1.1 and 1.96 respectively with mean PI 1.53

and no pregnancy was reported when endometrial thickness was lower than $7 \mathrm{~mm}$.

In this study, the best cut-off value of resistance index (RI) for predicting increased uterine blood flow impedance was RI more than 0.67 , with sensitivity of $100 \%$, specificity of $96.7 \%$, positive predictive value of $97.5 \%$, negative predictive value of $100 \%$, and diagnostic accuracy of $98.6 \%$.

In the current study, the best cut-off value of pulsatility index (PI) for predicting increased uterine blood flow impedance was PI more than 1.95 , with sensitivity of $95 \%$, specificity of $86.7 \%$, positive predictive value of $90.47 \%$, negative predictive value of $92.86 \%$, and diagnostic accuracy of $91.42 \%$.

In this study, the best cut-off value of systolic/diastolic (S/D) ratio for predicting increased uterine blood flow impedance was S/D ratio more than 3 , with sensitivity of $100 \%$, specificity of $96.7 \%$, positive predictive value of
$97.56 \%$, negative predictive value of $100 \%$, and diagnostic accuracy of $98.57 \%$.

The current study results were in agreement with Hashad et al.'s study; they reported that best cut-off value of resistance index (RI) and pulsatility index (PI) for predicting increased uterine blood flow impedance was more than 0.67 and 1.37 respectively with sensitivity of both RI and PI being $70 \%$ and 77.5 respectively, specificity of both RI and PI $75 \%$ and $85 \%$ respectively, positive predictive value of both RI and PI $85 \%$ and $87 \%$ respectively, negative predictive value of both RI and PI $60 \%$ and $66 \%$ respectively, and diagnostic accuracy of both RI and PI $69.7 \%$ and 85.3 respectively [8].

This study concluded that the mean values of uterine artery Doppler indices including the resistance index (RI), the pulsatility index (PI), and the systolic/ diastolic (S/D) ratio were significantly higher in the

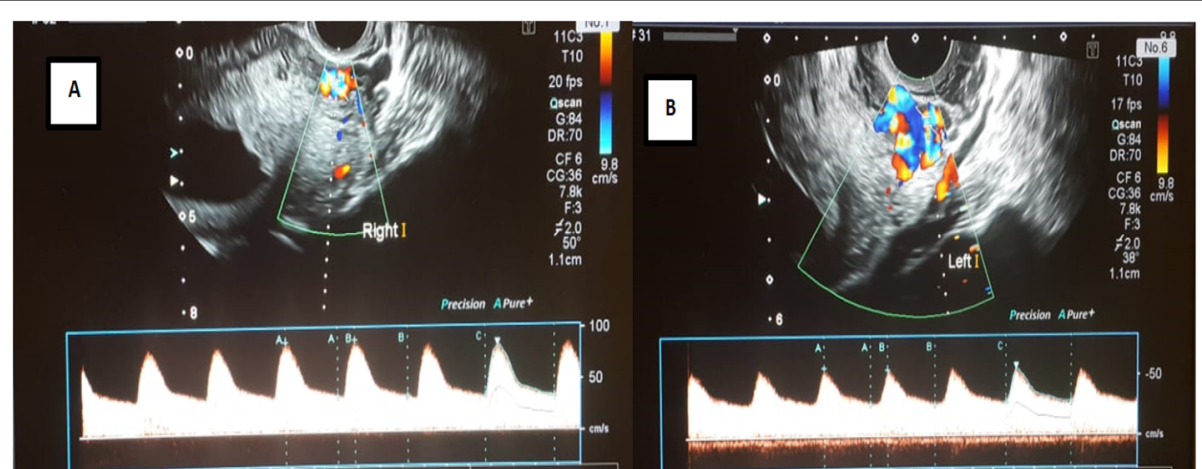

Fig. 5 a, b Transvaginal Doppler ultrasound of both uterine arteries (a right, $\mathbf{b}$ left) of fertile female (control group) aged 28 years old shows RI of right and left uterine arteries were 0.61 and 0.53 respectively with mean RI 0.57, S/D ratios of right and left uterine arteries were 2.59 and 2.1 respectively with mean S/D ratio 2.34, and PI of right and left uterine arteries were 0.95 and 0.75 respectively with mean PI 0.85 . 


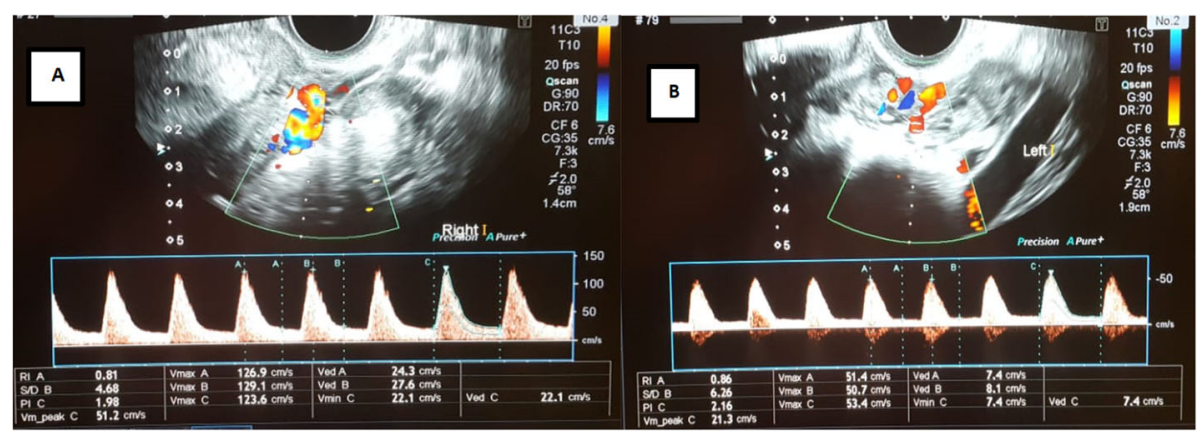

Fig. $\mathbf{6}$ a, b Transvaginal Doppler ultrasound of both uterine arteries (a right, $\mathbf{b}$ left) of female patient aged 29 years old diagnosed with unexplained infertility shows Rl of right and left uterine arteries were 0.8 and 0.86 respectively with mean RI 0.83 , S/D ratios of right and left uterine arteries were 4.68 and 6.26 respectively with mean S/D ratio 5.47, and PI of right and left uterine arteries were 1.98 and 2.16 respectively with mean PI 2.07

study (infertile) group than the control (fertile) group with significant difference in the endometrial blood flow impedance and uterine perfusion in both study and control groups.

Uterine artery Doppler with measurement of Doppler indices including the resistance index (RI), the pulsatility index (PI), and the systolic/diastolic (S/D) ratio provides high sensitivity and specificity with high diagnostic accuracy for diagnosis of high uterine artery blood flow impedance in women diagnosed with unexplained infertility.

This study concluded that the uterine blood flow impedance was significantly higher in women that were diagnosed with unexplained infertility (study group) more than fertile women (control group), so high uterine blood flow impedance and low endometrial perfusion diagnosed by uterine artery Doppler may contribute in the etiology of unexplained infertility.

This study is recommended to include the uterine artery Doppler in the investigations of unexplained infertility.

This study is recommended to study a large numbers of female diagnosed with unexplained infertility and study the effect of drugs that increase uterine perfusion for treatment of unexplained infertility and increase the possibility of pregnancy.

\section{Conclusion}

Uterine artery Doppler indices mean values were higher in unexplained infertility women than fertile women. Uterine artery Doppler indices have high sensitivity and

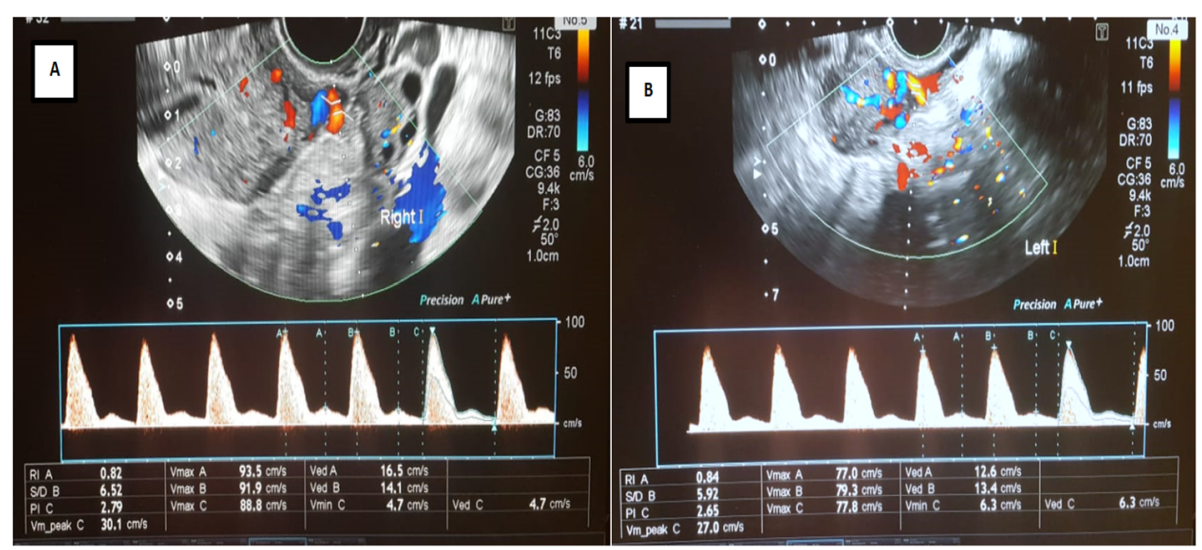

Fig. 7 a, b Transvaginal Doppler ultrasound of both uterine arteries (a right, $\mathbf{b}$ left) of female patient aged 31 years old diagnosed with unexplained infertility shows RI of right and left uterine arteries were 0.82 and 0.84 respectively with mean RI 0.83, S/D ratios of right and left uterine arteries were 6.52 and 5.92 respectively with mean S/D ratio 6.22, and PI of right and left uterine arteries were 2.79 and 2.65 respectively with mean PI 2.72 


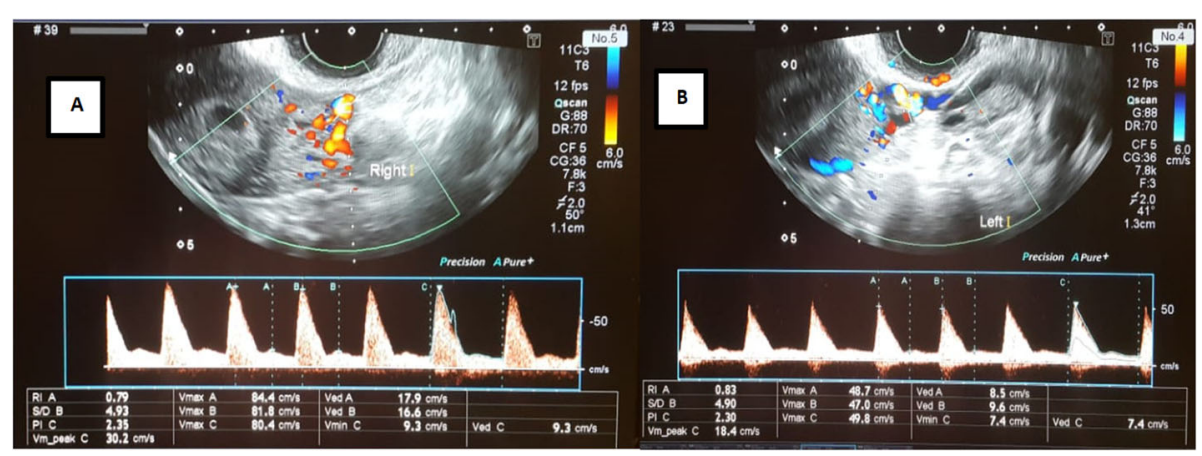

Fig. 8 a, b Transvaginal Doppler ultrasound of both uterine arteries (a right, $\mathbf{b}$ left) of female patient aged 33 years old diagnosed with unexplained infertility shows RI of right and left uterine arteries were 0.79 and 0.83 respectively with mean RI 0.81, S/D ratios of right and left uterine arteries were 4.93 and 4.9 respectively with mean S/D ratio 4.91, and PI of right and left uterine arteries were 2.35 and 2.3 respectively with mean PI 2.32

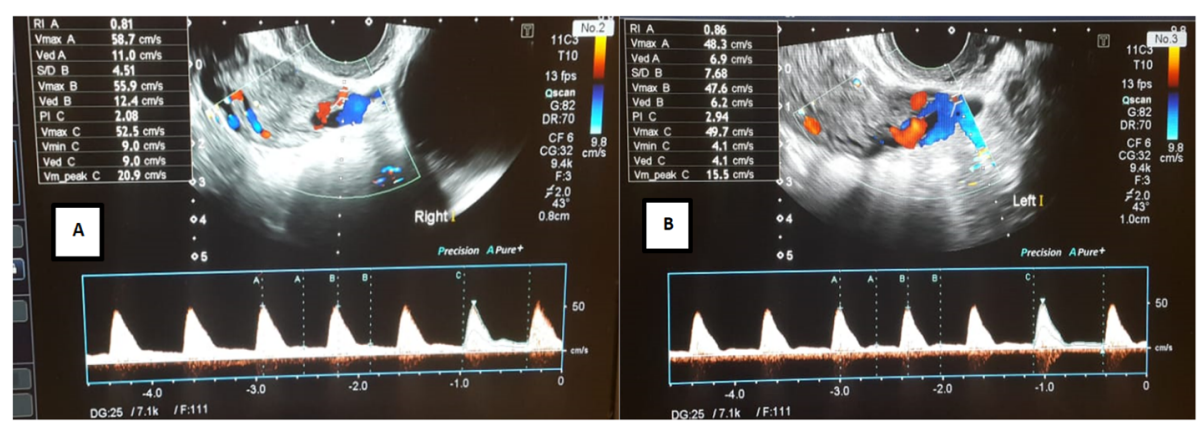

Fig. 9 a, b Transvaginal Doppler ultrasound of both uterine arteries (a right, $\mathbf{b}$ left) of female patient aged 26 years old diagnosed with unexplained infertility shows RI of right and left uterine arteries were 0.81 and 0.86 respectively with mean RI 0.83, S/D ratios of right and left uterine arteries were 4.51 and 7.68 respectively with mean S/D ratio 6.09, and PI of right and left uterine arteries were 2.08 and 2.94 respectively with mean PI 2.51

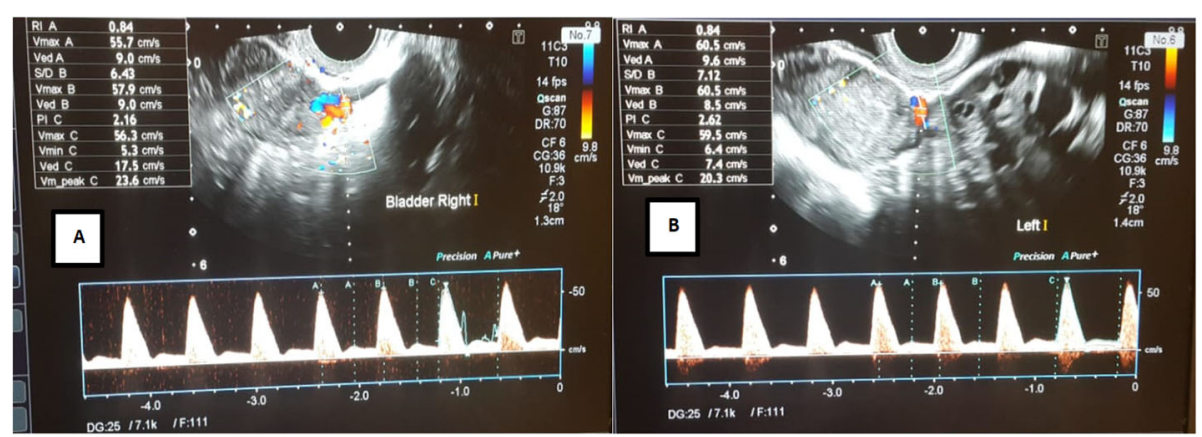

Fig. 10 a, b Transvaginal Doppler ultrasound of both uterine arteries (a right, $\mathbf{b}$ left ) of female patient aged 33 years old diagnosed with unexplained infertility shows RI of right and left uterine arteries were 0.84 with mean RI 0.84 , S/D ratios of right and left uterine arteries were 6.43 and 7.12 respectively with mean S/D ratio 6.77, and PI of right and left uterine arteries were 2.16 and 2.62 respectively with mean PI 2.39 
specificity for diagnosis of high uterine blood flow impedance. High uterine blood flow impedance diagnosed by uterine artery Doppler may contribute in the etiology of unexplained infertility. Uterine artery Doppler should be included in the investigation of unexplained infertility.

\section{Abbreviations}

RI: Resistance index; PI: Pulsatility index; S/D: Systolic/diastolic; SD: Standard deviation; IVF: In vitro fertilization; IVF-ET: In vitro fertilization-embryo transfer; IUI: Intrauterine insemination

\section{Acknowledgements}

Not applicable.

\section{Authors' contributions}

CAZ and WSA carried out the work. CAZ designed the study. WSA collected the patients and gathered the data. CAZ and WSA collected and reported the radiological data. CAZ did the statistical analysis and was responsible for collecting the scientific data and writing the manuscript. All authors read and approved the final version to be published.

\section{Funding}

Not applicable.

\section{Availability of data and materials}

All data and material of the article are readily available.

\section{Ethics approval and consent to participate}

The authors obtained permission to conduct this study and was approved by Research Ethic Committee (REC) at Faculty of Medicine Port Said University, Port Said, Egypt (serial number: (2) RAD), and all participants gave written informed consent. The procedures followed were in accordance with our protocol.

\section{Consent for publication}

All participants gave written informed consent.

\section{Competing interests}

The authors declare that they have no competing interests

\section{Author details}

1Diagnostic Radiology, Faculty of Medicine, Port Said University, Port Said Egypt. ${ }^{2}$ Gynecology and Obstetrics, Faculty of Medicine, Port Said University, Port Said, Egypt.

Received: 7 October 2020 Accepted: 1 February 2021

Published online: 18 February 2021

References

1. Pandey S, Khanna G, Bajpai A, Khanna A (2014) Emerging role of color Doppler in infertility management: a public health perspective. Fertil Sci Res 1:87-91

2. Fenning NJ, Campbell BK, Kendall NR et al (2004) Endometrial and subendometrial perfusion are impaired in women with unexplained subfertility. Human Reprod 19:2605

3. El-Shourbagy S, Ossman AME, El-Mohamady A (2017) The role of sildenafil citrate (viagra) suppositories on endometrial response (thickness and mean resistance index of endometrial spiral artery) in cases of unexplained infertility. Menoufia Med J 30:343-349

4. El-Toukhy T, Coomarasamy A, Khairy M, Sunkara K et al (2008) The relationship between endometrial thickness and outcome of medicated frozen embryo replacement cycles. Fertil Steril 89:832-839

5. Ng EH, Chan CC, Tang OS, Yeung WS, Ho PC (2007) The role of endometrial blood flow measured by three-dimensional power Doppler ultrasound in the prediction of pregnancy during in vitro fertilization treatment. Eur J Obstet Gynecol Reprod Biol 135:8-16

6. Edi-Osagie EC, Seif MW, Aplin JD et al (2004) Characterizing the endometrium in unexplained and tubal factor infertility: a multiparametric investigation. Fertil Steril 82:1379-1389
7. Adibi A, Khadem M, Mardanian F, Hovsepian S (2012) Uterine and arcuate arteries blood flow for predicting of ongoing pregnancy in in vitro fertilization. J Res Med Sci: Off J Isfahan Univ Med Sci 20(9):879

8. Hashad AM, Ibrahim IM, Etman MK et al (2018) A study of endometrial perfusion in unexplained infertility. Egyptian J Hosp Med. 72(6):4673-4680

9. Devyatova EA, Tsaturova KA, Vartanyan EV (2016) Predicting of successful implantation at IVF cycles. Gynecol Endocrinol 32(2):27-29

10. Gong X, LI Q, Zhang Q et al (2005) Predicting endometrium receptivity with parameters of spiral artery blood flow. J Huazhong Univ Sci Technol 25:335-338

11. The Practice Committee of the American Society for Reproductive Medicine, authors (2006). Effectiveness and treatment for unexplained infertility. Fertil Steril; 86: S111-S114.

12. Chien LW, Au HK, Chen PL et al (2002) Assessment of uterine receptivity by the endometrial-subendometrial blood flow distribution pattern in women undergoing in vitro fertilization-embryo transfer. Fertil Steril 78:245-251

13. Uysal S, Özün Özbay EP, Ekinci T, Aksüt H, Karasu S, Işık AZ, Soylu F (2012) Endometrial spiral artery Doppler parameters in unexplained infertility patients: is endometrial perfusion an important factor in the etiopathogenesis? J Turk Ger Gynecol Assoc 13:169-171

14. El-Mazny A (2016) Doppler ultrasonography in infertility and assisted reproduction. Austin J In Vitro Fertili 3(1):1028

15. Selda U, Elif P, Tekin E et al (2012) Endometrial spiral artery Doppler parameters in unexplained infertility patients: is endometrial perfusion an important factor in the etiopathogenesis? J Turkish-German Gynecol Assoc 13:169-171

16. Makhija K, Shrivasatava D, Tiwari M (2019) Predictive value of uterine artery: peak systolic velocity on the day of trigger for clinical pregnancy rate in infertile women. Int J Reprod, Contracept, Obstet Gynecol 8(2):677-681

17. Prasad S, Goyal R, Kumar Y, Nayar P et al (2017) The relationship between uterine artery two-dimensional color doppler measurement and pregnancy outcome: a prospective observational study. J Reproduct Infertil 18(2):251

18. Guzman ER, Kontopoulos E and Zalud I (2005): Doppler velocimetry of the uteroplacental circulation. In: Doppler Ultrasound in Obstetrics and Gynecology. 2nd edi; Dev Maulik (ed).

19. Chien LW, Lee WS, Au HK, Tzeng CR (2004) Assessment of changes in utero ovarian arterial impedance during the peri implantation period by Doppler sonography in women undergoing assisted reproduction. Ultrasound Obstet Gynecol 23:496-500

20. Cacciatore B, Simberg N, Fusaro P et al (1996) Transvaginal Doppler study of uterine artery blood flow in in vitro fertilization-embryo transfer cycles. Ferti Steril 66:130-134

21. Ari K, Tae K, Sang W (2010) Relationship between endometrial and subendometrial blood flow measured by three dimensional power Doppler ultrasound and pregnancy after intrauterine insemination. Fertil Steril 94 747-752

22. Khan MS, Shaikh A, Ratnani R (2016) Ultrasonography and Doppler study to predict uterine receptivity in infertile patients undergoing embryo transfer. J Obstet Gynecol India 66(S1):S377-S382

23. Noyes N, Hampton BS, Berkeley A et al (2001) Factors useful in predicting the success of oocyte donation: a 3-year retrospective analysis. Fertil Steril 76(1):92-97

24. Kovacs P, Matyas S, Boda K et al (2003) The effect of endometrial thickness on IVF/ICSI outcome. Hum Reprod 18(11):2337-2341

\section{Publisher's Note}

Springer Nature remains neutral with regard to jurisdictional claims in published maps and institutional affiliations. 